\title{
Transonic flow calculations using a dimensional splitting method
}

\author{
Hing Hung John A. Gear Natalie J. T. Phillips*
}

(Received 7 August 2000)

\begin{abstract}
The time-dependent Transonic Small Disturbance equation, will be reformulated in Hyperbolic Conservation Law form. In Hyperbolic Conservation Law form, with dimensional splitting the equations can be discretized and treated as a series of Riemann problems. Steady subsonic and transonic flow calculations will be presented for a suitable test configuration and where possible comparisons will be made with analytic solutions.
\end{abstract}

*Department of Mathematics, RMIT University, Melbourne, VIC 3001, AustraliA.

${ }^{0}$ See http: //anziamj . austms . org. au/V42/CTAC99/Hung for this article and ancillary services, (C) Austral. Mathematical Soc. 2000. Published 27 Nov 2000. 


\section{Contents}

1 Introduction

C753

2 Governing Equations

C755

3 Numerical Methods

C757

3.1 FVS applied to the $z$ equation . . . . . . . . . . . C760

3.2 Riemann Problem for the TSD Equation. . . . . . . . . . C761

4 Boundary Conditions

C765

5 Numerical Results

C767

6 Conclusion

$\mathrm{C} 770$

References

C772

\section{Introduction}

For over fifty years it has been known that wings and control surfaces of high speed aircraft are most unstable (aeroelastically), at transonic speeds. The Transonic Small Disturbance (TSD) Equation [2] of transonic flow and its approximations, is a common model equation for describing subsonic and 
supersonic flow close to the local speed of sound (transonic). The velocity potential and hence the pressure (on the bounding surfaces of an aircraft), can be determined from the solution of the TSD equation. In transonic flow there is an embedded region of locally supersonic flow inside an otherwise subsonic flow. Usually a shock discontinuity terminates the supersonic region. Recent advances in numerical techniques have made possible the calculation of transonic flow over realistic structures $[2,3,4,5]$. The success of a numerical scheme for transonic flow prediction depends on its capability of capturing all the flow details while keeping the cost of computation low.

Our aim here is to develop numerical methods for the solution of the TSD equation, written in Hyperbolic Conservation Law form. In Hyperbolic Conservation Law form, the TSD equation can be recast as coupled Riemann Problems (one in each dimension) using dimensional splitting. These Riemann Problems are then solved by employing one of various one dimensional finite volume methods. In this way the solution to be determined for flow over a general multi-dimensional geometry can be obtained independent of the meshing. Initially the TSD equation will be used to model the steady subsonic flow over aerofoils for which the exact solution is known. Finally, the result of steady transonic flow calculations over the NACA 0012 aerofoil will be presented. 


\section{Governing Equations}

In two space dimensions the Transonic Small Disturbance (TSD) equation for the perturbation velocity potential $\phi[2]$ may be written in the form

$$
\frac{\partial}{\partial t}\left(\phi_{x}+\phi_{t}\right)+\frac{\partial}{\partial x}\left(\phi_{t}+\frac{\beta^{2}}{2 \bar{u} M^{2}}\left(\phi_{x}-\bar{u}\right)^{2}\right)-\frac{1}{M^{2}} \frac{\partial}{\partial z} \phi_{z}=0,
$$

where $M$ is the free stream Mach number, and $\beta$ and $\bar{u}$ are given by

$$
\beta^{2}=1-M^{2} \quad \text { and } \quad \bar{u}=\beta^{2} /\left(M^{2}(1+\gamma)\right),
$$

where $\gamma$ is the ratio of specific heats. Here $(x, z)$ represents a nondimensional Cartesian coordinate system, $x$ streamwise and $z$ vertical, and $t$ is a nondimensional time variable. In nondimensional terms the fluid velocity is $\mathbf{v}=\nabla(x+\phi)$. Equation (1) is locally hyperbolic representing supersonic flow for $\phi_{x}>\bar{u}$ and elliptic representing subsonic flow for $\phi_{x}<\bar{u}$.

If the upper and lower surface of the wing is defined as $z=h^{ \pm}(x, t)$, then the wing flow tangency condition is

$$
\phi_{z}^{ \pm}=\left\{\begin{array}{lll}
h_{x}^{ \pm}+h_{t}, & z=0 & \text { unsteady flow } \\
h_{x}^{ \pm}, & z=0 & \text { steady flow. }
\end{array}\right.
$$

The + and - superscript indicate the upper and lower wing surfaces. Across the wake (behind the wing) there can be no jump in pressure or normal 
velocity. In terms of the perturbation potential these conditions are:

$$
\begin{array}{lll}
\left\langle\phi_{z}\right\rangle=0 & \text { on } z=0, & \\
\left\langle\phi_{x}+\phi_{t}\right\rangle=0 & \text { on } z=0 & \text { unsteady flow, } \\
\left\langle\phi_{x}\right\rangle=0 & \text { on } z=0 & \text { steady flow. }
\end{array}
$$

Equation (1) can be written as a system of first order equations by defining

$$
u=\phi_{x}, \quad w=\phi_{z}, \quad p=\phi_{x}+\phi_{t} \quad \text { and } \quad \mathbf{u}=(u, w, p)^{T}
$$

and assuming $\phi_{x z}=\phi_{z x}$, giving in Conservation Law Form

$$
\frac{\partial \mathbf{u}}{\partial t}+\frac{\partial}{\partial x} \mathbf{F}(\mathbf{u})+\frac{\partial}{\partial z} \mathbf{H}(\mathbf{u})=0
$$

$$
\text { with } \begin{aligned}
\mathbf{F}(\mathbf{u}) & =\left(u-p, w, p-u+\frac{\beta^{2}}{2 \bar{u} M^{2}}(u-\bar{u})^{2}\right)^{T}, \\
\mathbf{H}(\mathbf{u}) & =\left(0,-p,-\frac{w}{M^{2}}\right)^{T} .
\end{aligned}
$$

Equation (3) can be expressed in quasilinear conservation law form as

$$
\frac{\partial \mathbf{u}}{\partial t}+\mathbf{A}(\mathbf{u}) \frac{\partial \mathbf{u}}{\partial x}+\mathbf{B}(\mathbf{u}) \frac{\partial \mathbf{u}}{\partial z}=0
$$

where $\mathbf{A}$ and $\mathbf{B}$ are the Jacobian matrices of $\mathbf{F}$ and $\mathbf{H}$ respectively,

$$
\mathbf{A}(\mathbf{u})=\left(\begin{array}{ccc}
1 & 0 & -1 \\
0 & 1 & 0 \\
-1+\frac{\beta^{2}}{\bar{u} M^{2}}(u-\bar{u}) & 0 & 1
\end{array}\right), \quad \mathbf{B}(\mathbf{u})=\left(\begin{array}{ccc}
0 & 0 & 0 \\
0 & 0 & -1 \\
0 & -\frac{1}{M^{2}} & 0
\end{array}\right)
$$


On the wing the flow tangency condition Equation (2) becomes

$$
w^{ \pm}=h_{x}^{ \pm}+h_{t}, \quad \frac{\partial u^{ \pm}}{\partial z}=h_{x x}^{ \pm}+h_{x t}, \quad{\frac{\partial p^{ \pm}}{\partial z}}^{ \pm}=h_{x x}^{ \pm}+h_{x t}+h_{t t}
$$

where for steady flow $\frac{\partial}{\partial t}=0$ and on the wake $\langle w\rangle=0$ and $\langle p\rangle=0$.

A numerical solution of Equations (4) needs to be found on a finite computational grid. At the outer boundaries of the grid suitable non-reflective conditions must be applied. On the upstream and downstream boundaries the appropriate far field conditions are

$$
u=\frac{\partial w}{\partial x}=p=0 .
$$

On the upper and lower boundaries the appropriate farfield conditions are

$$
\frac{\partial u}{\partial z}=w=\frac{\partial p}{\partial z}=0
$$

\section{$3 \quad$ Numerical Methods}

Let time $t$ be discretized such that $t_{n}=n \Delta t$ for $n=1,2, \ldots$ where $\Delta t$ is the discrete time increment and the solution at $\left(x, z, t_{n}\right)$ is $\mathbf{u}^{n}$. On each time interval $t_{n} \leq t \leq t_{n+1}$ we have a two dimensional initial value problem (IVP)

$$
\begin{array}{ll}
\mathrm{PDE} & \mathbf{u}_{t}+\mathbf{F}(\mathbf{u})_{x}+\mathbf{H}(\mathbf{u})_{z}=0 \\
\mathrm{IC} & \mathbf{u}\left(x, z, t_{n}\right)=\mathbf{u}^{n}
\end{array}
$$


giving $\mathbf{u}\left(x, z, t_{n+1}\right)$ when solved. It is convenient, computationally, to solve this as a sequence of one dimensional IVPs using dimensional splitting. That is if $t$ is the local variable $t \in[0, \Delta t]$, IVP (5) is approximated by the one dimensional IVPs

$$
\begin{array}{cc}
\mathbf{u}_{t}^{*}+\mathbf{F}\left(\mathbf{u}^{*}\right)_{x}=0 \\
\mathbf{u}^{*}(x, z, 0)=\mathbf{u}\left(x, z, t_{n}\right) \\
\text { and } \quad \begin{array}{c}
\mathbf{u}_{t}+\mathbf{H}(\mathbf{u})_{z}=0 \\
\mathbf{u}(x, z, 0)=\mathbf{u}^{*}(x, z, 0)
\end{array}
\end{array}
$$

here $\mathbf{u}^{*}$ denotes the temporal solution.

Let $\mathcal{L}_{x}(\Delta t)$ denote the solution operator for the $x$ equation in time increment of $\Delta t$ and $\mathcal{L}_{z}(\Delta t)$ for the $z$ equation, then a first order approximation in time of $\mathbf{u}\left(x, z, t_{n+1}\right)$ is

$$
\begin{aligned}
\mathbf{u}\left(x, z, t_{n+1}\right) & =\mathcal{L}_{x}(\Delta t) \mathcal{L}_{z}(\Delta t) \mathbf{u}(x, z, 0), \\
& =\mathcal{L}_{x}(\Delta t) \mathcal{L}_{z}(\Delta t) \mathbf{u}^{n}
\end{aligned}
$$

representing first solving the $z$ equation followed by the solution of the $x$ equation. A second order approximation, which is computationally more expensive, is

$$
\mathbf{u}\left(x, z, t_{n+1}\right)=\mathcal{L}_{x}(\Delta t / 2) \mathcal{L}_{z}(\Delta t) \mathcal{L}_{x}(\Delta t / 2) \mathbf{u}^{n} .
$$

The order of the operations does not affect the accuracy of the schemes but may affect computational efficiency. Dimensional Splitting schemes are constructed using Taylor series expansions [1] which rely on the smoothness of 
u. Nonsmooth solutions may cause dimensional splitting schemes to fail depending on the numerical method applied to each one dimensional equation. Generally, schemes that smear discontinuities are most successful [6]. For this reason we chose the Godonov method for (6) and Flux Vector Splitting (FVs) for (7). Both of these methods are conservative methods which for a general system of $m$ one dimensional hyperbolic equations

$$
\mathbf{u}_{t}+\mathbf{F}(\mathbf{u})_{x}=0
$$

with real and distinct eigenvalues $\lambda_{i}$ for $i=1,2, \ldots, m$, is of the form

$$
\mathbf{u}_{i}^{n+1}=\mathbf{u}_{i}^{n}+\frac{\Delta t}{\Delta x}\left(\mathbf{F}_{i-\frac{1}{2}}^{n}-\mathbf{F}_{i+\frac{1}{2}}^{n}\right) .
$$

The space domain $[0, L]$ has been discretised into $I$ computing cells $I_{i}=$ $\left[x_{i-\frac{1}{2}}, x_{i+\frac{1}{2}}\right]$ of regular size $\Delta x=\frac{L}{I}$ with cell centre at $x_{i}$ for $i=1,2, \ldots, I$. $\mathbf{u}_{i}$ represents the approximation of $\mathbf{u}\left(x_{i}\right)$ and $\mathbf{F}_{i+\frac{1}{2}}^{n}$ is the numerical intercell flux at position $x_{i+\frac{1}{2}}$ and time $t_{n}$.

For the Godonov method $\mathbf{F}_{i+\frac{1}{2}}^{n}=\mathbf{F}^{n}\left(\mathbf{u}_{i+\frac{1}{2}}(0)\right)$, where $\mathbf{u}_{i+\frac{1}{2}}(0)$ is the solution evaluated at $x=0$ of $\operatorname{RP}(i, i+1)$, the local Riemann Problem with initial states $\mathbf{u}_{i}$ and $\mathbf{u}_{i+1}$. For FVs methods $\mathbf{F}_{i+\frac{1}{2}}^{n}=\mathbf{F}^{+}\left(\mathbf{u}_{\mathbf{i}}^{\mathbf{n}}\right)+\mathbf{F}^{-}\left(\mathbf{u}_{i+1}^{n}\right)$ where $\mathbf{F}^{+}$ and $\mathbf{F}^{-}$are components of $\mathbf{F}$ such that $\mathbf{F}=\mathbf{F}^{+}+\mathbf{F}^{-}$. If $\mathbf{F}$ satisfies the homogeneity property $\mathbf{F}=\mathbf{A u}, \mathbf{A}$ is the Jacobian matrix of $\mathbf{F}$, then $\mathbf{F}=$ $\mathbf{A}^{+} \mathbf{u}+\mathbf{A}^{-} \mathbf{u}$ where $\mathbf{A}^{+}=\mathbf{P} \Lambda^{+} \mathbf{P}^{-1}$ and $\mathbf{A}^{-}=\mathbf{P} \Lambda^{-} \mathbf{P}^{-1}$ so that $\lambda_{i}^{+} \geq 0$ and $\lambda_{i}^{-} \leq 0$ form the diagonal of the diagonal matrices $\Lambda^{+}$and $\Lambda^{-}$respectively, 
and $\lambda_{i}=\lambda_{i}^{+}+\lambda_{i}^{-}$for $i=1,2, \ldots, m$. If (8) is linear, (9) becomes

$$
\mathbf{u}_{i}^{n+1}=\mathbf{u}_{i}^{n}+\frac{\Delta t}{\Delta x}\left[\mathbf{A}^{+}\left(\mathbf{u}_{i+1}-\mathbf{u}_{i}\right)+\mathbf{A}^{-}\left(\mathbf{u}_{i}-\mathbf{u}_{i+1}\right)\right] .
$$

This represents a simple downwind differencing of the $z$ derivatives for $\lambda_{i}^{+}$ and upwind differencing for $\lambda_{i}^{-}$. This simple technique is found to give stable schemes for scalar PDEs. The stability of both methods is determined by

$$
S_{\max }^{n} \frac{\Delta t}{\Delta x} \leq 1
$$

where $S_{\max }^{n}$ is the maximum wave speed over the space domain at $t_{n}[6]$.

\subsection{FVS applied to the $z$ equation}

We apply the FVS method to the linear IVP (7) with $\mathbf{H}$ defined as in Section 2. Using the Steger and Warming definition of $\lambda_{i}^{+}$and $\lambda_{i}^{-}$

$$
\lambda_{i}^{+}=\frac{1}{2}\left(\lambda_{i}+\left|\lambda_{i}\right|\right) \quad \text { and } \quad \lambda_{=}^{-} \frac{1}{2}\left(\lambda_{i}-\left|\lambda_{i}\right|\right)
$$

where for $\mathbf{H} \lambda_{1}=-\frac{1}{M}, \lambda_{2}=0$ and $\lambda_{3}=\frac{1}{M}$, gives a numerical scheme first order in time and $z$

$$
\mathbf{u}_{j, k}^{*}=\mathbf{u}_{j, k}^{n}-\frac{\Delta t}{\Delta z}\left(\mathbf{B}^{+}\left(\mathbf{u}_{j, k}^{n}-\mathbf{u}_{j, k-1}^{n}\right)+\mathbf{B}^{-}\left(\mathbf{u}_{j, k+1}^{n}-\mathbf{u}_{j, k}^{n}\right)\right),
$$


for $i=1,2, \ldots, I$ and $k=2,3 \ldots, K-1$, and

$$
\mathbf{B}^{+}=\left(\begin{array}{ccc}
0 & 0 & 0 \\
0 & \frac{1}{2 M} & -\frac{1}{2} \\
0 & -\frac{1}{2 M^{2}} & \frac{1}{2 M}
\end{array}\right) \quad \text { and } \quad \mathbf{B}^{-}=\left(\begin{array}{ccc}
0 & 0 & 0 \\
0 & -\frac{1}{2 M} & -\frac{1}{2} \\
0 & -\frac{1}{2 m^{2}} & -\frac{1}{2 M}
\end{array}\right) \text {. }
$$

Scheme (10) is stable, when $\Delta t$ and $\Delta z$ are chosen such that $\max _{i}\left|\lambda_{i}\right| \frac{\Delta t}{\Delta z} \leq 1$.

\subsection{Riemann Problem for the TSD Equation.}

The discretization of $t$ and the space domain into $I$ computing cells $I_{i}, i=$ $1,2, \ldots, I$, creates a series of local Riemann Problems, whose $i$ th term is

$$
\mathbf{u}(x, 0)=\left\{\begin{array}{lr}
\mathbf{u}_{t}+\mathbf{F}(\mathbf{u}) x=0 \\
\mathbf{u}_{j+1, k}^{n}=\mathbf{u}_{L}=\mathbf{u}_{R} & \text { if } x>0
\end{array}\right.
$$

Here $t$ and $x$ are taken to be the local variables $t \in[0, \Delta t]$ and $x \in\left[-\frac{\Delta t}{2}, \frac{\Delta t}{2}\right]$. Following [6], we note that the eigenvalues of $\mathbf{A}$ are $\lambda_{1}=1-\sqrt{1-\alpha(u-\bar{u})}$, $\lambda_{2}=1$ and $\lambda_{3}=1+\sqrt{1-\alpha(u-\bar{u})}$ with corresponding eigenvectors $k_{1}=$ $(1,0, \sqrt{1-\alpha(u-\bar{u})})^{T}, k_{2}=(0,1,0)^{T}$ and $\left.k_{3}=(1,0,-\sqrt{1-\alpha(u-\bar{u}})\right)^{T}$, where $\alpha=\frac{\beta^{2}}{\bar{u} M^{2}}=1+\gamma$. Of these $\lambda_{2}$ is a linearly degenerate characteristic field representing a contact discontinuity in the solution of $w$ with wave speed $\lambda_{2}=1 . \lambda_{1}$ and $\lambda_{3}$ are genuinely nonlinear characteristic fields [1] giving two 
possible wave types - shock waves and rarefaction waves. Across a shock wave the Rankine Hugoniot condition $\Delta \mathbf{F}=\lambda_{S} \Delta \mathbf{u}, \lambda_{S}$ is the shock speed, and the entropy condition $\lambda_{L} \leq \lambda_{S} \leq \lambda_{R}$ are satisfied, while across a rarefaction wave the generalised Riemann invariants

$$
\frac{d u}{k_{i}^{1}}=\frac{d w}{k_{i}^{2}}=\frac{d p}{k_{i}^{3}},
$$

where $k_{i}^{j}, j=1,2,3$ are the components of the eigenvector $k_{i}, i=1,2,3$, and the entropy condition $\lambda_{R} \geq \lambda_{L}$ apply. Therefore we need to consider four possible wave formations. If $k_{1}$ is a rarefaction wave the generalised Riemann invariants imply $w$ is constant and

$$
p^{*}+\frac{2}{3 \alpha}\left(1-\alpha\left(u^{*}-\bar{u}\right)\right)^{\frac{3}{2}}=p_{L}+\frac{2}{3 \alpha}\left(1-\alpha\left(u_{L}-\bar{u}\right)\right)^{\frac{3}{2}} .
$$

Where $*$ represents the "star" region [6] in which $\mathbf{u}$ is unknown. The entropy condition gives $u^{*} \geq u_{L}$, a condition for determining rarefaction. The speed of the wave fan is represented by the shock head $\lambda_{H}=1-\sqrt{1-\alpha\left(u^{*}-\bar{u}\right)}$ and the shock tail $\lambda_{T}=1-\sqrt{1-\alpha\left(u_{L}-\bar{u}\right)}$. If $\lambda_{1}$ is a shock wave then the Rankine Hugoniot condition gives

$$
p^{*}=p_{L}+\sqrt{1-\alpha(\overline{\bar{u}}-\bar{u}))}\left(u^{*}-u_{L}\right)
$$

$\overline{\bar{u}}=\frac{1}{2}\left(u^{*}+u_{L}\right)$ and the entropy condition requires $u^{*} \leq u_{L}$. The shock speed is then $\lambda_{S}=1-\sqrt{1-\alpha(\overline{\bar{u}}-\bar{u})}$. By a similar process, for $\lambda_{3}$

$$
p^{*}= \begin{cases}p_{R}+\frac{2}{3 \alpha}\left(1-\alpha\left(u^{*}-\bar{u}\right)\right)^{\frac{3}{2}}+\frac{2}{3 \alpha}\left(1-\alpha\left(u_{R}-\bar{u}\right)\right)^{\frac{3}{2}} & \text { if } \quad u^{*}>u_{R} \\ p_{R}-\sqrt{1-\alpha(\bar{u}-\bar{u}))}\left(u^{*}-u_{R}\right) & \text { if } \quad u^{*} \leq u_{R}\end{cases}
$$


with $\overline{\bar{u}}=\frac{1}{2}\left(u^{*}+u_{R}\right)$ and

$$
\begin{cases}\lambda_{H}=1-\sqrt{1-\alpha\left(u_{R}-\bar{u}\right)}, & \lambda_{T}=1-\sqrt{1-\alpha\left(u^{*}-\bar{u}\right)} \\ \lambda_{S}=1-\sqrt{1-\alpha(\bar{u}-\bar{u})} & \text { rarefaction, } \\ \text { shock. }\end{cases}
$$

$u^{*}$ and $p^{*}$ are then the solution of the equation formed by the combinations of waves. Defining

$$
f(u, v)= \begin{cases}-(u-v) \sqrt{1-\alpha(\overline{\bar{u}}-\bar{u})} & \text { if } u<v \\ \frac{2}{3 \alpha}\left[(1-\alpha(u-\bar{u}))^{\frac{3}{2}}-(1-\alpha(v-\bar{u}))^{\frac{3}{2}}\right] & \text { if } u>v,\end{cases}
$$

we then have $p_{R}-p_{L}+f\left(u^{*}, u_{L}\right)+f\left(u^{*}, u_{R}\right)=0$, a nonlinear equation in $u^{*}$. This equation can be solved with any of various techniques, but since $f(u, v)$ is monotone with non zero derivative, Newton's method is most efficient. So the complete solution to the Riemann Problem is a combination of the following:

1. for $\lambda_{1}$

(a) shock $\lambda_{S}=1-\sqrt{1-\alpha(\overline{\bar{u}}-\bar{u})}-u^{*} \leq u_{L}$

$$
\mathbf{u}(x, t)= \begin{cases}\left(u_{L}, w_{L}, p_{L}\right)^{T} & \text { if } \frac{x}{t} \leq \lambda_{S} \\ \left(u^{*}, w_{L}, p^{*}\right)^{T} & \text { if } \lambda_{S} \leq \frac{x}{t} \leq \lambda_{2}\end{cases}
$$

(b) rarefaction $-u^{*} \geq u_{L}$

$$
\mathbf{u}(x, t)= \begin{cases}\left(u_{L}, w_{L}, p_{L}\right)^{T} & \text { if } \frac{x}{t} \leq \lambda_{T} \\ \left(u_{f a n}, w_{L}, p_{f a n}\right)^{T} & \text { if } \lambda_{T} \leq \frac{x}{t} \leq \lambda_{H} \\ \left(u^{*}, w_{L}, p^{*}\right)^{T} & \text { if } \lambda_{H} \leq \frac{x}{t} \leq \lambda_{2}\end{cases}
$$


where $\lambda_{H}=1-\sqrt{1-\alpha\left(u^{*}-\bar{u}\right)}$ and $\lambda_{T}=1-\sqrt{1-\alpha\left(u_{L}-\bar{u}\right)}$, and

$$
u_{f a n}=\bar{u}+\frac{1}{\alpha}\left[1-\left(1-\frac{x}{t}\right)^{2}\right] \quad \text { and } \quad p_{f a n}=p_{L}-f\left(u_{f a n}, u_{l}\right)
$$

2. for $\lambda_{3}$

(a) shock with speed $\lambda_{S}=1+\sqrt{1-\alpha(\overline{\bar{u}}-\bar{u})}-u^{*} \leq u_{R}$

$$
\mathbf{u}(x, t)= \begin{cases}\left(u^{*}, w_{R}, p^{*}\right)^{T} & \text { if } \lambda_{2} \leq \frac{x}{t} \leq \lambda_{S} \\ \left(u_{R}, w_{R}, p_{R}\right)^{T} & \text { if } \lambda_{S} \leq \frac{x}{t}\end{cases}
$$

(b) rarefaction $-u^{*} \geq u_{R}$

$$
\begin{gathered}
\mathbf{u}(x, t)= \begin{cases}\left(u^{*}, w_{R}, p^{*}\right)^{T} & \text { if } \lambda_{2} \leq \frac{x}{t} \leq \lambda_{T} \\
\left(u_{f a n}, w_{R}, p_{f a n}\right)^{T} & \text { if } \lambda_{T} \leq \frac{x}{t} \leq \lambda_{H} \\
\left(u_{R}, w_{r}, p_{R}\right)^{T} & \text { if } \lambda_{H} \leq \frac{x}{t}\end{cases} \\
\lambda_{H}=1+\sqrt{1-\alpha\left(u_{R}-\bar{u}\right)} \text { and } \lambda_{T}=1+\sqrt{1-\alpha\left(u^{*}-\bar{u}\right)} \text { and } \\
u_{f a n}=\bar{u}+\frac{1}{\alpha}\left[1-\left(\frac{x}{t}-1\right)^{2}\right], \quad p_{f a n}=p_{L}-f\left(u_{f a n}, u_{l}\right) .
\end{gathered}
$$


The Godonov flux is evaluated at $x=0$ so only the first part of the solution applies, i.e..

$$
\mathbf{u}(0)=\left\{\begin{array}{ll}
\left(u_{L}, w_{L}, p_{L}\right)^{T} & \text { if } 0 \leq \lambda_{S} \\
\left(u^{*}, w_{L}, p^{*}\right)^{T} & \text { if } \lambda_{S} \leq 0 \leq \lambda_{2} \\
\left(u_{L}, w_{L}, p_{L}\right)^{T} & \text { if } 0 \leq \lambda_{T} \\
\left(u_{F a n}, w_{L}, p_{\text {Fan }}\right)^{T} & \text { if } \lambda_{T} \leq 0 \leq \lambda_{H} \\
\left(u^{*}, w_{L}, p^{*}\right)^{T} & \text { if } \lambda_{H} \leq 0
\end{array}\right\} \quad \text { if } u^{*} \geq u_{L}
$$

For scheme (9) applied to IVP (6) we have the iterative procedure

$$
\mathbf{u}_{i, k}^{n+1}=\mathbf{u}_{i, k}^{*}+\frac{\Delta t}{\Delta x}\left[\mathbf{F}_{i-\frac{1}{2}, k}^{*}-\mathbf{F}_{i+\frac{1}{2}, k}^{*}\right],
$$

for $i=2,3, \ldots, I-1$ and $k=2,3, \ldots, K-1$, where the initial data states for the local Riemann Problem $\operatorname{RP}(i, i+1)$ are $\mathbf{u}_{i, k}^{*}$ and $\mathbf{u}_{i+1, k}^{*}$.

The Godonov scheme is stable if $S_{\max }^{n} \frac{\Delta t}{\Delta x} \leq 1$. This requires that the wave speeds be predetermined or that $\Delta t$ be determined at each iteration. We chose the former approach with a conservative estimate $\Delta t=0.001$.

\section{Boundary Conditions}

To implement the boundary conditions an aerofoil is placed on the $x$ axis of a finite flow domain $[-1,2] \times[-1,1]$. The aerofoil shape is analytically 
continued onto the $x$ axis and represented as a boundary of infinitesimal width for $x \in[0,1]$. The flow domain is split into two blocks an upper block with wing surface $z=h^{+}(x)$ and a lower block with wing surface $z=h^{-}(x)$. On each block is placed a uniform grid with horizontal and vertical increments of $\Delta x$ and $\Delta z$. The vertical grid lines are placed such that the singularity at the leading and trailing edges lie on the midpoint of two lines. The wing surface lies on the midpoint of horizontal grid lines $k=1$ and $k=2$, where $k=1$ is a dummy grid level introduced to facilitate implementation of the boundary conditions and $k=2$ corresponds to $z=\frac{\Delta z}{2}$ for the upper block. The lower block is the mirror image of the upper block.

Applying a second order differencing to the $z$ derivative and averaging $w$ across $k=1$ and $k=2$, the flow tangency conditions become

$$
w_{i, 1}^{ \pm}=2 h_{x}^{ \pm}-w_{i, 2}^{ \pm}, \quad u_{i, 1}^{ \pm}=u_{i, 2}^{ \pm} \mp \Delta z h_{x x}^{ \pm} \quad \text { and } \quad p_{i, 1}^{ \pm}=p_{i, 2}^{ \pm} \mp \Delta z h_{x x}^{ \pm}
$$

where the superscripts + and - represent the upper and lower blocks. On the wake and diaphragm the zero jump conditions lead to

$$
w_{i, 1}^{ \pm}=w_{i, 2}^{\mp}, \quad u_{i, 1}^{ \pm}=u_{i, 2}^{\mp} \quad \text { and } \quad p_{i, 1}^{ \pm}=p_{i, 2}^{\mp}
$$

where in all cases $i=1,2, \ldots, I$. For streamwise and far vertical boundaries, the non-reflective conditions become

$$
\begin{array}{r}
u_{1, k}^{ \pm}=p_{1, k}^{ \pm}=u_{I, k}^{ \pm}=p_{I, k}^{ \pm}=0, w_{1, k}^{ \pm}=w_{2, k}^{ \pm}, w_{I, k}^{ \pm}=w_{I-1, k}^{ \pm}, \\
\quad \text { and } \quad w_{i, K}^{ \pm}=0, u_{i, K}^{ \pm}=u_{i, K-1}^{ \pm} \text {and } p_{i, K}^{ \pm}=p_{i, K-1}^{ \pm} .
\end{array}
$$

Where a first order one sided difference was applied to derivative conditions. 
TABLE 1: This table summarises the configuration of each test cases.

\begin{tabular}{|c|c|c|c|c|}
\hline test No & aerofoil shape & $M$ & $\Delta x$ & $\Delta z$ \\
\hline 1 & parabolic arc $10 \%$ thick & 0.5 & 0.033 & 0.033 \\
2 & flat plate $\theta=\tan ^{-1}(-.1)$ & 0.3 & 0.033 & 0.033 \\
3 & flat plate $\theta=\tan ^{-1}(-.1)$ & 0.5 & 0.033 & 0.033 \\
4 & NACA 0012 & 0.3 & 0.033 & 0.01 \\
5 & NACA 0012 & 0.82 & 0.02 & 0.01 \\
\hline
\end{tabular}

\section{$5 \quad$ Numerical Results}

In this section we present the results of applying schemes (11) and (14) successively with the boundary conditions in Section 4 and $\gamma=1.4$ to test configurations summarised in Table 1. In test case 1, a symmetric aerofoil of parabolic arc of $10 \%$ thickness, described by $h^{ \pm}(x)= \pm 0.2 x(1-x)$, is immersed in a flow field with free stream Mach number $M=0.5$. The grid spacing was chosen to be 0.033 in both directions a balance between accuracy and computational efficiency. The flow is characterised by symmetry about the $x$ axis and the line $x=0.5$ and an analytic expression for pressure coefficient $C_{p}$ on the wing surface is given by $C_{p}=(2+(-1+2 x) \ln (-1+1 / x)) /\left(5 \pi \sqrt{1-M^{2}}\right)$. This is obtained using a linear approximation of the TSD equation. For small $M$, the linear theory and nonlinear theory should give similar results. In Figure 1 the negative pressure coefficient $-C_{p}$ obtained by the numerical scheme, represented by the dots, is compared to the analytic solution (un- 


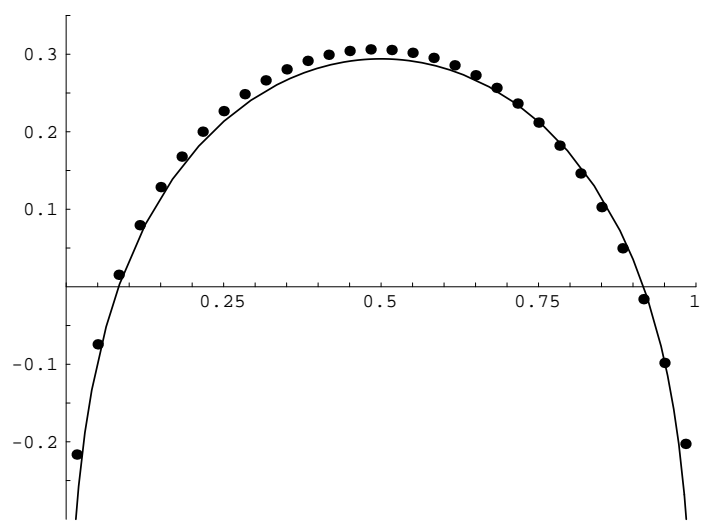

Figure 1: Negative of Pressure coefficient on upper surface of symmetric $10 \%$ thick parabolic aerofoil at $M=0.5$.

broken line). The approximation of subsonic flow by the TSD equation results in skewness in the solution. This may be attributable to the nonlinearity in the TSD equation. In test case 2 , we have an antisymmetric flow over a flat plate inclined at an angle $\theta$ such that $\tan \theta=-0.1$ and free stream Mach number of 0.3 . The wing surface is represented by $h^{ \pm}(x)=-0.1(x-0.5)$. Again the grid spacing was chosen to be 0.033 in both directions. In test case 3 , the flat plate of test 2 is placed in a flow field with $M=0.5$ and the grid configuration is unaltered. Figures $2(\mathrm{a})$ and $2(\mathrm{~b})$ show the result of test cases 2 and 3 compared with an analytic expression for $-C_{p}$ found using a linear approximation of the TSD equation. For test 2 the approximation is 


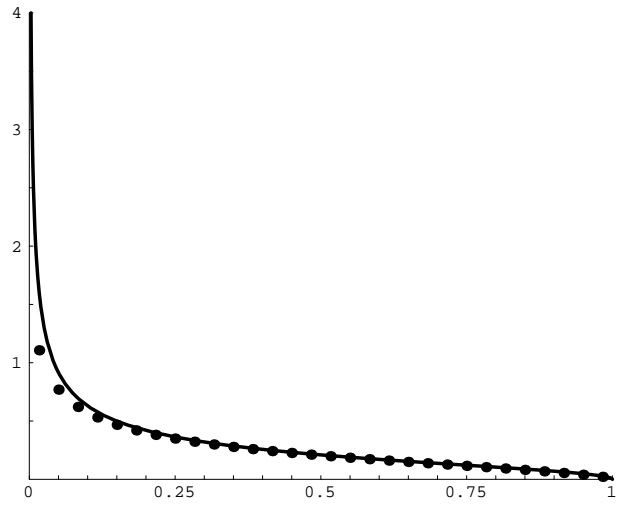

(a)

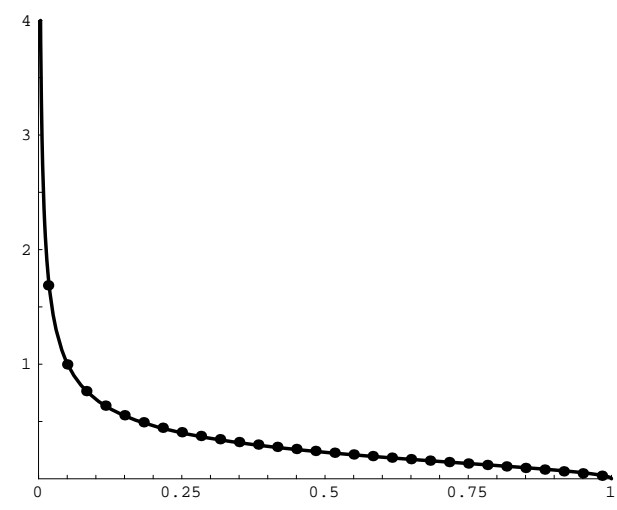

(b)

Figure 2: Negative of pressure coefficient on the upper surface of flat plate inclined at $\tan \theta=-0.1$ for $M=0.3$ in (a) and $M=0.5$ in (b).

worst near the leading edge but gave accurate solution elsewhere. In Figure 3 we see a good agreement between the analytic and numerical solutions. The TSD equation was formulated with the assumption that $M$ be close to 1 , we have used it to approximate subsonic flow where $M$ is small. Therefore it should be expected that as $M$ increases then the approximation becomes more accurate.

Figure 3(a) compares, for test 4, numerical results obtained by different numerical methods, dots for our scheme and unbroken line for the spectral method. In test case 4, we have the more practical NACA 0012 aerofoil 
with $M=0.3$ and grid spacing of 0.033 in the $x$ direction and 0.01 in the $z$ direction. Again the greatest error lies near the singularity at the leading edge, where for better approximation requires a finer meshing in both $x$ and $z$ directions. In test case 5, the NACA 0012 aerofoil is subjected to free stream flow of $M=0.82$. At this Mach number a shock develops and for better resolution of the shock the $x$ increment was reduced to 0.02 and the $z$ increment to 0.01 . The results of test 5 are summarised in Figure 3(b), where the spectral method is represented by the unbroken line and our scheme by dots. In terms of shock resolution our scheme is better as a spectral method gives an overshoot in the shock. In both schemes the shock is resolved within 3 grid points. In all cases except test 5 the solution was presented at the end of 5000 iterations. This is excessively inefficient in terms of computation time, but may be explained by the very conservative choice for $\Delta t=0.001$ and that the Godonov and FVs methods are explicit first order. In test 5 the plotted solution was after 10000 iterations, this is due to the smaller $\Delta x$ and $\Delta z$.

\section{Conclusion}

The nonlinear TSD equation was reformulated in hyperbolic conservation law form and with the aid of dimensional splitting, is solved using the one dimensional finite volume methods of Godonov and flux vector splitting. While the solution for steady subsonic flow is accurate in general, there are 


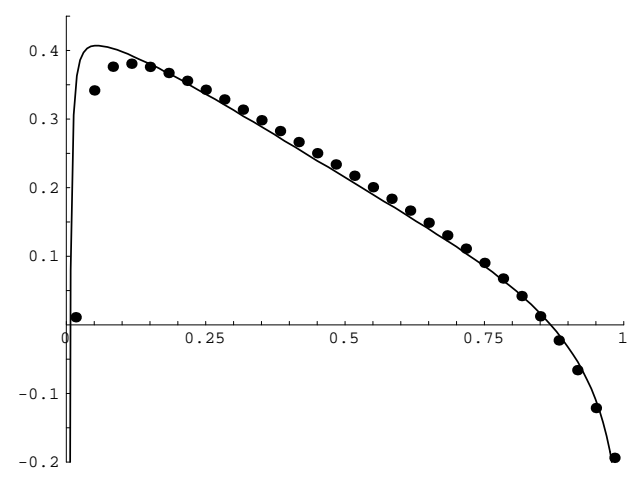

(a)

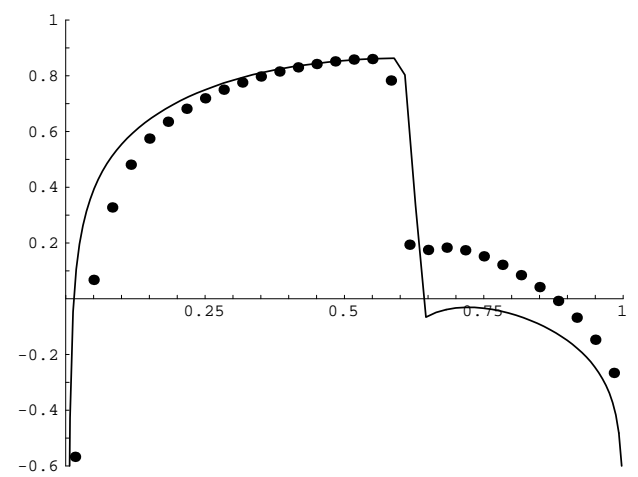

(b)

Figure 3: Negative of pressure coefficient on the upper surface of flat plate inclined at $\tan \theta=-0.1$ for $M=0.3$ in (a) and $M=0.5$ in (b). 
inaccuracies near the leading edge. This can be partially rectified by refining the mesh but in terms of computational efficiency, this is not a viable option. Nevertheless, more importantly, the success or failure of a numerical scheme applied to the TSD equation depends on the quality of the solution around shocks. In this regard, our scheme appears to be successful. Still the scheme requires much improvements. We propose in future work to apply the method of time step cycling to enhance the rate of convergence.

\section{References}

[1] R. J. Le Veque. Numerical Methods for Conservation Laws. Birkhauser, Berlin, 1990. C758, C761

[2] J. T. Batina. Efficient algorithm for solution of the unsteady transonic small-disturbance equation. J. Aircraft, 25:598-605, 1988. C753, C754, C755

[3] J. A. Gear and F. G. Polanco. An approximate factorization algorithm for the unsteady transonic small disturbance equation. in R.L. May and A.K. Easton, editors, Computational Techniques and Application: CTAC95, pages 335-342, Singapore, 1995. World Scientific. C754

[4] G. P. Guruswamy and P. M. Goorjian. An efficient coordinate transformation technique for unsteady, transonic aerodynamic analysis of low aspect-ratio wings. AIAA, paper-84-0872, 1984. C754 
[5] J. A. Gear, E. Ly, and N. J. T. Phillips. Time marching finite difference solution of the modified transonic small disturbance equation. In J. Noye, M. Teubner, and A. Gill, editors, Computational Techniques and Applications: CTAC97, pages 209-216, Singapore, 1998. World Scientific. C754

[6] E. F. Toro. Riemann Solvers and Numerical Methods for Fluid Dynamics: A Practical Introduction. Springer Verlag, Berlin, 1997. C759, C760, C761, C762 